\title{
Stage I Gastric and Omental Gastrointestinal Stromal Tumor AJCC v8
}

National Cancer Institute

\section{Source}

National Cancer Institute. Stage I Gastric and Omental Gastrointestinal Stromal Tumor

A/CC v8. NCl Thesaurus. Code C136770.

Stage I includes: IA: (T1 or T2, N0, M0, Low Mitotic Rate); IB: (T3, N0, M0, Low Mitotic Rate). T1: T umor measuring $2 \mathrm{~cm}$ or less. T2: T umor measuring more than $2 \mathrm{~cm}$ but not more than $5 \mathrm{~cm}$. T3: T umor measuring more than $5 \mathrm{~cm}$ but not more than $10 \mathrm{~cm}$. N0: No regional lymph node metastasis or unknown lymph node status. M0: No distant metastasis. Low Mitotic Rate: Five or fewer mitoses per 5 square millimeters, or per 50 HPF. (AJCC 8th ed.) 